\section{The introduction of verteporfin photodynamic therapy in the UK: PDT users group (PDTUG) surveillance programme report 1}

FD Ghanchi ${ }^{1}$, J Fullarton ${ }^{2}$, J Blake ${ }^{2}$ and SP Harding ${ }^{3}$ on behalf of the PDTUG(UK)

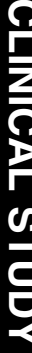

\section{Abstract}

Aims To report overall patient recruitment characteristics and visual acuity (VA) outcome related to baseline lesion characteristics for patients with choroidal neovascularisation (CNV) treated with verteporfin photodynamic therapy (PDT) during its introduction into routine National Health Service practice.

Methods Thirteen treatment centres prospectively submitted data on patients undergoing verteporfin PDT for CNV of mixed aetiology between November 1999 and May 2004 into the PDT Users Group (PDTUG) surveillance database. The primary outcome was the proportion of eyes losing $<15$ letters of VA at 12 and 24 months, follow-up compared with the baseline examination. Results One thousand eight hundred and ninety-four eyes of $\mathbf{1 7 5 5}$ patients were analysed. Lesion characteristics at baseline were: classic no occult $1152(67.4 \%)$, predominantly classic with occult 531 (31.1\%). Recruitment rate rose steadily from 13 in the first to $\mathbf{1 8 8}$ in the final quarter. Data were available at 12 months on $1010(53.3 \%)$ and at 24 months on $310(16.4 \%)$ eyes. The proportion of eyes losing < 15 letters was $71 \%$ (716/1010) at 12 months and $70 \%(217 / 310)$ at 24 months. At 12 months 91\% (917/1010) of patients lost $<30$ letters. The mean number of PDT treatments for the cohort was 2.4 in the first 12 months. An adverse reaction or event was reported in $8.1 \%(364 / 4515)$ of treatments. Non-visual adverse events were infrequent. Conclusions Efficacy and safety of verteporfin PDT in reducing vision loss in macular degeneration can be reproduced in routine clinical practice. Compared to the TAP study, the fewer treatments needed in the PDTUG cohort indicate the potential for better cost-effectiveness.

Eye (2008) 22, 671-677; doi:10.1038/sj.eye.6702713; published online 16 March 2007

Keywords: choroidal neovascularisation; age-related macular degeneration; verteporfin photodynamic therapy; visual acuity; effectiveness and safety

\section{Introduction}

Subfoveal choroidal neovascularisation (CNV) is the major cause of visual loss in patients with age-related macular degeneration (AMD) accounting for $90 \%$ of all registered blindness owing to AMD. ${ }^{1}$ The development of neovascularisation from the choriocapillaris often results in irreversible degeneration of the overlying neurosensory retina, leading to loss of visual acuity (VA) and deterioration of contrast sensitivity (CS). ${ }^{2} \mathrm{CNV}$ is defined by the proportions of subtypes of CNV (classic, occult) to each other and other lesion components visible on fluorescein angiography, ${ }^{3}$ as well as its location (extrafoveal, juxtafoveal, subfoveal). ${ }^{4}$ Recent epidemiological studies ${ }^{5,6}$ estimate that $3.6 \%$ of the UK population aged 75 years or older are visually impaired owing to AMD. Vision loss is more likely to be severe in patients with subfoveal lesions compared to extrafoveal ${ }^{7}$ and can lead to a profound reduction in quality of life. ${ }^{8}$

Historically, effective treatment for CNV secondary to AMD has been limited. ${ }^{9}$ Laser photocoagulation treatment has been shown to
${ }^{1}$ Department of Ophthalmology, Bradford Teaching Hospitals NHS Foundation Trust, Royal Infirmary, Bradford, UK

${ }^{2}$ Strategen Ltd, 2 \& 3 Stable Court, Herriard, Basingstoke, UK

${ }^{3}$ St Paul's Eye Unit, Royal Liverpool University Hospital, Prescot St, Liverpool, UK

Correspondence: FD

Ghanchi, Department of Ophthalmology,

Bradford Teaching Hospitals NHS Foundation Trust,

Royal Infirmary,

Duckworth Lane,

Bradford,W Yorkshire, BD9 6RJ, UK

Tel: + 4401274364117 Fax: + 4401274366710. E mail: faruque.ghanchi@ bradfordhospitals.nhs.uk

Received: 18 June 2006 Accepted in revised form: 30 November 2006; Published online: 16 March 2007 
be beneficial in some patients ${ }^{10}$ but the immediate loss of vision after treatment of subfoveal $\mathrm{CNV}^{11}$ means that it is typically restricted to extrafoveal lesions. Two multicentre, randomised controlled clinical trials, the treatment of age-related macular degeneration with Photodynamic Therapy (TAP) study ${ }^{10,12}$ and Verteporfin in Photodynamic Therapy (VIP) study, ${ }^{13,14}$ have shown that photodynamic therapy (PDT) with verteporfin (Visudyne $^{\mathrm{TM}}$, Novartis) significantly reduces the risk of visual loss. PDT is the current treatment of choice for patients with subfoveal CNV with a range of alternative therapies and regimes in development.

In 1999, the UK PDT Users Group (PDTUG) national surveillance programme was established to monitor visual response in routine clinical practice outside a research study and to provide further safety data. Preliminary reports from the surveillance programme facilitated the National Institute for Clinical Excellence (NICE) consultation process, which published guidance on the use of PDT in the National Health Service (NHS) for AMD in 2003. ${ }^{15}$ For the UK, treatment is currently restricted to subfoveal CNV owing to any cause with a VA of $\geqslant 6 / 60$ and predominantly classic with or without occult.

This paper reports on the visual outcomes observed in patients treated with verteporfin PDT based on prospectively collected data for audit under the PDTUG surveillance programme.

\section{Materials and methods}

All patients commencing a course of verteporfin PDT for CNV for any aetiology in 13 UK clinical treatment centres (Appendix 1) were followed prospectively within the PDTUG surveillance programme. Patients were required to fulfill eligibility criteria determined by the treating ophthalmologist as shown in Table 1. The steering committee considered that recruitment to a placebo-controlled group was unethical based on the

Table 1 Principal eligibility criteria for entry into the PDT users group surveillance programme

\begin{tabular}{l}
\hline Inclusion criteria \\
\hline Subfoveal and juxtafoveal CNV secondary to any cause \\
Subfoveal and juxtafoveal CNV secondary to pathological \\
myopia with a history of recent visual loss \\
Classic with no occult or predominantly classic with occult \\
$\mathrm{VA} \geqslant 35$ letters (Snellen equivalent $6 / 60$ ) \\
Exclusion criteria \\
Lesions within $1000 \mu$ m of maximum laser spot size \\
$<50 \%$ classic $\mathrm{CNV}$, retinal pigment epithelium (RPE) tears
\end{tabular}

published results from previous randomised controlled studies. Patients were followed for up to 24 months.

The study was designed as an audit of the introduction of a new technology into clinical practice and was deemed as such by the Liverpool Adult Research Ethics Committee. Consent for the collection of data was collected as part of the normal consent process for treatment and according to local rules in each centre.

\section{Vision testing}

After refraction, VA was tested by a retinal specialist or trained vision assessor using the modified Early Treatment Diabetic Retinopathy Study (ETDRS) vision chart at baseline, 12 and 24 months. Test distance was $2 \mathrm{~m}$ except where less than 20 letters were read in which case re-testing was performed on the top three lines at $1 \mathrm{~m}$. Best-corrected VA was calculated as the total number of letters seen correctly at $1 \mathrm{~m}$.

\section{Angiographic classifications}

Classification of lesion characteristics and aetiology was performed locally at each treatment centre by a retinal specialist. CNV was defined as subfoveal if present under and juxtafoveal if within $200 \mu \mathrm{m}$ of the centre of the foveal avascular zone.

\section{Verteporfin PDT}

Treatment was applied according to the recommendations of the $\mathrm{TAP}^{10,12}$ and $\mathrm{VIP}^{13,14}$ studies. Verteporfin $\left(6 \mathrm{mg} / \mathrm{m}^{2}\right.$ of body surface area) was administered by intravenous infusion in a volume of $30 \mathrm{ml}$ over $10 \mathrm{~min}$. At $15 \mathrm{~min}$ after the start of the infusion, a $689 \mathrm{~nm}$ laser source was used to deliver $50 \mathrm{~J} / \mathrm{cm}^{2}$ over $83 \mathrm{~s}$ using a spot diameter $1000 \mu \mathrm{m}$ larger than the greatest linear diameter (GLD) of the CNV lesion.

\section{Patient follow-up}

In addition to the baseline examination, all patients were scheduled for follow-up visits every 3 months. At each scheduled follow-up visit, VA measurement, ophthalmoscopic examination and fundus fluorescein angiography were performed. The decision to retreat was made by the treating retinal specialist based on evidence of lesion activity (persisting or increased subretinal fluid, haemorrhage, new loss of vision, angiographic leakage) and likelihood of no further benefit (vision below 20 letters, primary non-response, or adverse event, development of chorioretinal anastomosis or retinal pigment epithelial tear). Adverse events considered by the treating retinal specialist to be drug related were 
recorded including: infusion-related (lower-back pain, injection site events), visual loss (transient, $\geqslant 20$ letters lost), ocular (RPE tear, intraocular haemorrhage), systemic (photosensitivity, other).

\section{Data monitoring and reporting}

Participating centres prospectively collected a minimum data set, anonymised to maintain patient confidentiality. No patient attributable information (name, address, contact details) was included in data transfer. Data were provided on a regular basis either electronically using custom made software, or paper-based on standard PDT surveillance forms and submitted to the coordinating centre (Liverpool) for entry onto the database software. The minimum data set included: visit date, lesion characteristics, aetiology, month since first treatment, VA, lesion size (GLD $(\mu \mathrm{m}))$, adverse events and reactions, treatment given and any deviation from treatment protocol.

\section{Outcome measurements}

The primary outcome measure was the response rate at 12 months, defined as the proportion of patients free from moderate vision loss ( $<15$ letters lost using ETDRS vs baseline). This is based on previous research in the field and represents doubling of the visual angle that is definitely noticeable to the patient. ${ }^{16}$ Other VA-related measures included the response rate at 12 and 24 months depending on GLD of lesion.

The data for the study were captured on a continuous basis as patients attended for regular clinic sessions. For a proportion of patients whose data for exactly timed 6-, 12-, or 24-month visits were missing, the data captured at the next quarterly visit were substituted or, if these were absent, the data since the previous quarterly visit were used.

\section{Statistical analysis}

Statistical analyses were carried out using the mathematical and statistical tools provided by Microsoft Access and SPSS for Windows. Treated eyes were defined as 'second eyes' where the fellow eye had vision of 45 letters or worse $(6 / 18)$.

\section{Results}

Between November 1999 and May 2004, data were collected from 1972 patients. Of these, 217 (11\%) had incomplete data or errors made in the records. Further inspection showed that the errors were non-systematic and the omission of these data would not bias the analysis of the properly completed records. This decision was supported by comparing the database before and after the omission of these records in terms of: mean age at the end of the study (74.96 vs 74.94 years); mean baseline VA (46.20 vs 46.64 letters); mean baseline lesion GLD (2198 vs $2246 \mu \mathrm{m}$ ); and sample prevalence of AMD where reported (75.68 vs $75.42 \%$ ). No statistical differences between these baseline parameters were detected and so, for the purposes of this report, data from 1755 patients were analysed. One hundred and thirty-nine patients received treatment to both eyes giving 1894 eyes included in the report. Of these 855 $(45.7 \%)$ were first eyes and $1016(54.3 \%)$ were second eyes; where in the first eye's visual acuity was less than $6 / 18$ (45 letters). Baseline characteristics of all registered eyes as determined by the treating retinal specialist are shown in Table 2. The majority (67.4\%) of eyes for which a lesion type was recorded had classic $\mathrm{CNV}$ with no occult. In the majority $(75.7 \%)$ of eyes where it was recorded, the aetiology was AMD. 53\% of the lesions (52.9\%) had a GLD of over $2000 \mu \mathrm{m}$ and $57 \%$ of eyes (1079/1894) had baseline VA of more than 45 letters.

At the end of the study period 1559 eyes had completed 6 months follow-up since registration, 1010 had completed 12 months follow-up since registration, and 310 had completed 24 months follow-up since registration. These figures are a reflection of continuous and accelerating recruitment over the study period and are shown in Figure 1. Thirteen eyes were recruited in the first compared to 188 in the final quarter. Over a 24-month period, 7502 eye/visits were carried out at 13 centres and involved a total of 4514 treatments. Drop-outs before the end of study in 2004 were infrequent; 54 patients did not reach 1 year follow-up, of which 36 were recorded as discharged. Similarly of the 90 patients who had not reached 24 months follow-up were 61 who had completed 12 months follow-up (52\% success

Table 2 Baseline characteristics of 1755 patients (1894 eyes) with $\mathrm{CNV}$

\begin{tabular}{lc}
\hline & No. (\% of recorded) \\
\hline CNV aetiology & \\
AMD only & $1246(75.7)$ \\
AMD (recurrence after laser treatment) & $116(7.0)$ \\
Other cause & $284(17.3)$ \\
Not recorded & $248(13.0)$ \\
& \\
Lesion type & \\
Classic/no occult & $1152(67.4)$ \\
Predominantly classic CNV & $531(31.1)$ \\
Minimally classic CNV & $10(0.6)$ \\
Occult/no classic & $17(1.0)$ \\
Not recorded & $184(9.7)$ \\
\hline
\end{tabular}




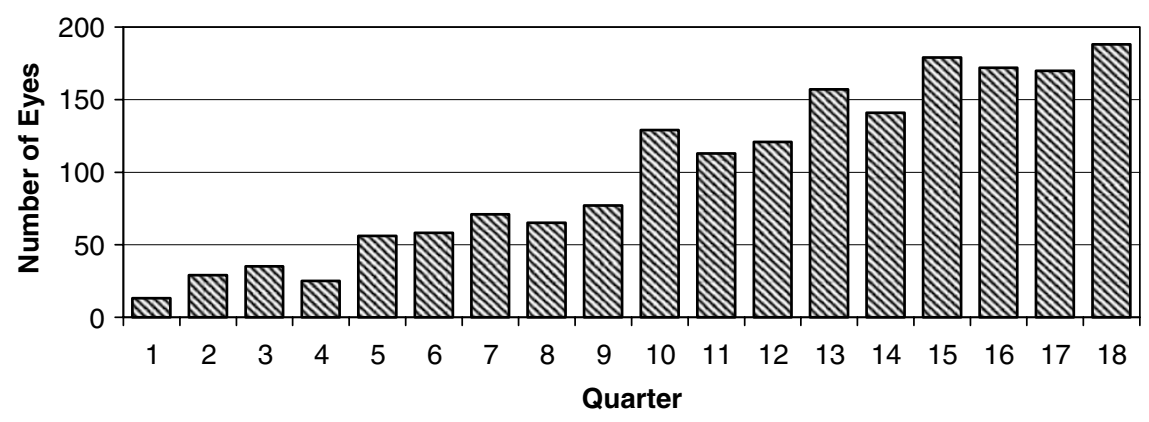

Figure 1 Recruitment rate per quarter during the study period.

Table 3 Change in vision (letters) and response rate for study eyes with AMD in PDTUG surveillance programme

\begin{tabular}{lccccc}
\hline Follow-up period & No. of eyes & Mean baseline VA & Mean 6 months VA & Responder rate n, (\%) & Mean change (letters) \\
\hline 6 months & 1411 & 48.37 & 41.59 & $1047(74.2 \%)$ & -6.78 \\
12 months & 905 & 49.31 & 41.93 & $643(71.0 \%)$ & -7.38 \\
24 months & 269 & 50.55 & 43.53 & $190(70.6 \%)$ & -7.02 \\
\hline
\end{tabular}

rate) and are included in the 1-year analysis. Reasons for drop-out in the remaining (29) patients, included severe adverse event (1), death (2), refused treatment (3), nonattendance (3), and discharge (20).

\section{Visual outcome}

Table 3 shows the visual outcome and responder rate for patients with classic/no occult or predominantly classic AMD patients reaching 6, 12, and 24 months of follow-up during the study period. In the AMD patients, prevention of moderate loss of vision ( $\geq 15$ letters lost) was achieved in $71.0 \%$ at 12 months and $70.6 \%$ at 24 months. The mean change in number of letters read from baseline was -7.38 at 12 months and -7.02 at 24 months. The 12 months response rate compared to baseline was better for predominantly classic lesions with occult when compared to the classic with no occult group (75 vs 69\%, Pearson $\chi^{2}$ test, $\left.P=0.03\right)$. Severe loss of vision $(\geqslant 30$ letters lost) was observed in $93(9.2 \%)$ of 1010 patients at 12 months follow-up and $27(8.7 \%)$ of 310 at 24 months follow-up.

In those patients in this mixed cohort for whom complete data are available for the required time period, there were an average 2.4 treatments in the first 12 months and 3.4 treatments in 24 months, that is, an average of 1.0 treatment in the second year.

\section{Safety}

Three hundred and fifty-eight (7.9\%) of 4514 treatment episodes were associated with a total of 396 adverse
Table 4 Frequency of individual adverse events in 4514 treatment episodes ${ }^{\mathrm{a}}$

\begin{tabular}{|c|c|c|}
\hline $\begin{array}{l}\text { Adverse reaction/ } \\
\text { event }\end{array}$ & $\begin{array}{c}\text { Number of } \\
\text { treatment episodes }\end{array}$ & $\begin{array}{c}\% \text { of treatment } \\
\text { episodes } \\
(\mathrm{n}=4514)(\%)\end{array}$ \\
\hline Allergic reaction $^{\mathrm{b}}$ & 2 & 0.04 \\
\hline Back pain & 132 & 2.92 \\
\hline CNS event (mild) & 15 & 0.33 \\
\hline Chest pain & 8 & 0.18 \\
\hline Extravasation & 9 & 0.20 \\
\hline Haemorrhage & 26 & 0.58 \\
\hline Irritation/rash & 4 & 0.09 \\
\hline Pain of injection & 4 & 0.09 \\
\hline Photosensitivity & 3 & 0.07 \\
\hline RPE tear & 8 & 0.18 \\
\hline Transient visual loss & 22 & 0.49 \\
\hline$>20$ letters lost & 137 & 3.04 \\
\hline Other ocular event & 15 & 0.33 \\
\hline $\begin{array}{l}\text { Other non-ocular } \\
\text { event }^{c}\end{array}$ & 9 & 0.20 \\
\hline
\end{tabular}

event $^{\mathrm{c}}$

aTreatment episodes may be associated with more than one adverse event.

binclude one case of allergic reaction to fluorescein before PDT treatment. 'Includes one case of tuberculosis which is unlikely to be related to therapy.

events. Table 4 shows the frequency of individual adverse events. Acute loss of vision ( $\geqslant 20$ letters) was associated with 137 (3.04\%) treatments. Infusion-related back pain occurred in $132(2.9 \%)$ and chest pain in eight $(0.18 \%)$ treatment episodes. Less frequently reported adverse events included transient visual loss, haemorrhage, photosensitivity, injection site pain, back pain, and extravasation. 
Two hundred and seventy-three (15.6\%) of 1755 patients, experienced at least one adverse event at some point in the course of treatment reported to the surveillance programme. Infusion-related back pain and chest pain occurred in $89(5.07 \%)$ and eight $(0.46 \%)$ patients, respectively, and $23(1.31 \%)$ patients suffered haemorrhage. Adverse reactions were generally transient during the study. One hundred and twenty-one patients (6.9\%) were reported to have $>20$ letter loss at least once during follow-up. However, of these patients, only two $(0.11 \%)$ suffered this loss of vision in the 7 days immediately following a PDT treatment, the decline in the majority of patients being more gradual. In $33 \%$ $(40 / 121)$ of these patients some vision was recovered within 9 months. Out of 310 patients who were followed for 24 months or more (rather than had an actual visit recorded at 24 months), 53 patients (17.1\%) experienced $>20$ letters lost, and 32 patients $(10.3 \%)$ experienced $>30$ letters lost compared to baseline. No deaths related to PDT were reported.

\section{Variation between clinical centres}

Numbers of eyes recruited into the database at each centre were: LIV 863, WLV 241, NEW 238, BRD 129, SHE 125, TOR 61, MAN 59, DOR 49, BAR 40, LEE 31, BAT 29, GRI 19, SUN 10. Centres started recruiting at different times ranging from $5 / 11 / 99$ to $6 / 6 / 03$. The variation in case mix across the 13 centres is shown in Figure 2 with AMD representing $60 \%$ or more of the aetiology at each participating centre except for Sunderland which provided the lowest number of cases.

Two centres with less than 20 eyes (GRI and SUN) were excluded from further comparative analysis. Over

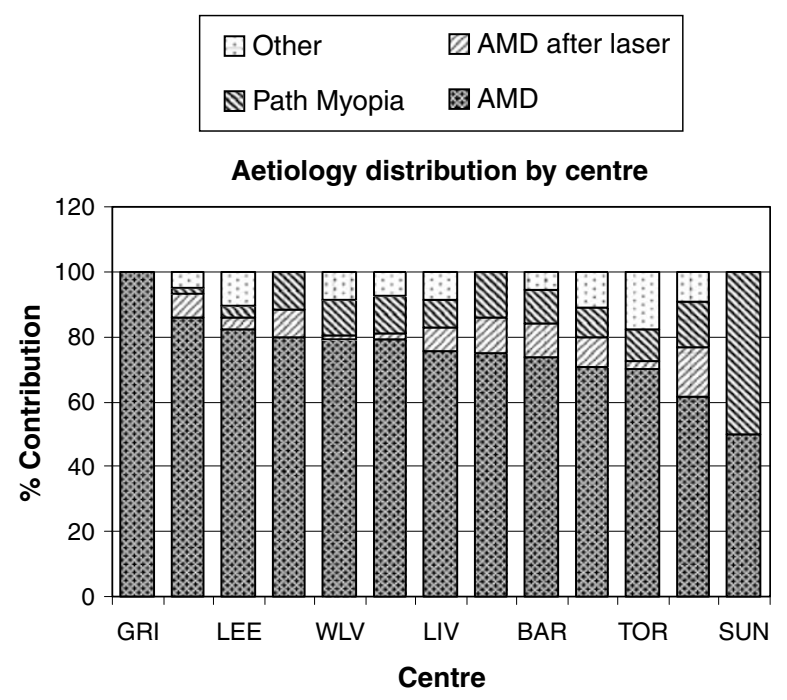

Figure 2 Aetiology distribution by centre. the first 12 months the proportion of eyes with $<15$ letters lost ranged between 59 and 100\% (mean 71\%). Although three centres recruited the majority of patients registered under the surveillance programme, the average response rate for these three centres was 70\%, in line with the average response rate for all the centres. The mean number of treatments required ranged between 1.4 and 3.2 (mean 2.4) and frequency of adverse events between 1.4 and 11.1 (mean 7.7). In the majority of centres, the proportion of classic/no occult lesions was in excess of $50 \%$ (mean $67.4 \%$ ) and the proportion of lesions diagnosed as AMD in the majority of centres was over $60 \%$ (mean $75.7 \%$ ). Overall the effectiveness of verteporfin PDT was good in all centres.

\section{Discussion}

The PDTUG surveillance programme was designed to collect information on the effectiveness and safety of verteporfin therapy from 13 UK centres providing a range of service configurations within the NHS. Seven thousand five hundred and two eye visits were carried out at 13 centres and a total of $4514(60.1 \%)$ verteporfin PDT treatments were applied. The patients studied under the PDTUG surveillance programme were a heterogeneous group of whom the majority $(75.7 \%)$ were patients with AMD. The results of AMD patients under the surveillance programme compare favourably with the TAP study, the published randomised controlled study which was used to demonstrate efficacy for licensing and approval for introduction by NICE. The mean loss of 7.4 letters at 12 months in our AMD patients compares favourably with a loss of 9.9 letters in TAP (predominantly classic and classic/no occult groups). ${ }^{10}$ It should be pointed out that among the PDTUG patients, $10.7 \%$ had juxtafoveal lesions which potentially have a more favourable outcome. Similarly the 12 month response rate of $71 \%$ (Table 3 ) compares favourably with the $67 \%$ reported in TAP. ${ }^{10}$ Similar to the TAP study most of the VA loss occurred in the first 12 months, especially during the first 3-6 months of therapy. ${ }^{12}$

There are a number of weaknesses in any post-licensing surveillance programme. In a formal RCT major resources are available to ensure robust data collection and full follow-up. Numbers of patients fell with longer follow-up in our programme with 53.3\% (1010/1894) of eyes reaching 12 months and 16.4\% (310/1894) reaching 24 months. This was mainly due to slow take up of verteporfin PDT across the UK in the period before NICE final appraisal determination. As the data collection and analysis was carried out at a stage of on going recruitment in routine clinical practice, relatively smaller numbers are available for analysis especially for longer follow-up. Additionally, the missing data on aetiology 
(13\%), number of patients lost to follow-up and some not reaching the time point for analysis (12 and 24 months) were compounding factors. On the other hand, the advantage of a surveillance programme such as ours is the large numbers and the ability to measure the effectiveness of a new treatment in routine clinical practice. Although the numbers at 24 months are small compared to at baseline our data suggest that the effect seen at 12 months was maintained at 24 months.

In the PDTUG surveillance programme the mean number of treatments over the 24-month follow-up at 3.4 was less than the 5.6 in the treatment arm of the TAP study. ${ }^{12}$ This reduction in the number of treatments has potentially favourable implications for the cost-effectiveness of verteporfin therapy.

It is recognised that the results are based on analysis of patients with neovascular macular degeneration that included pathologies other than AMD that can have better treatment outcome than AMD. The results of PDTUG surveillance programme may therefore overestimate the treatment response. The variation in case mix and uptake across centres may suggest differences in the way clinicians interpret data from randomised clinical trials and the influence of local commissioning policies. With the introduction of NICE guidelines it is hoped that there may be a reduction in variation and this will become apparent in future surveillance programmes. The rate of recruitment across the UK increased steadily over the 4.5 years surveyed.

For a number of centres recruitment was halted by local commissioning after a small number of patients had been recruited and did not recommence. If these few centres with short recruitment periods are disregarded then there was a good level of consistency across remaining centres for case mix and outcomes.

Verteporfin PDT was well tolerated, with few adverse events that were generally transient and mild to moderate in intensity. No deaths were reported and no patients stopped treatment because of an adverse event judged by the treating ophthalmologist to be related to verteporfin treatment. The type and incidence of adverse events reported in the PDTUG surveillance programme were similar to those reported in the TAP ${ }^{12}$ and VIP ${ }^{14}$ studies. In the PDTUG surveillance programme the overall frequency of adverse events was significantly lower than in the TAP study treatment arm. ${ }^{12}$ Infusion-related back pain was seen more frequently in the PDTUG surveillance programme compared to the TAP study ${ }^{12}$ (4.05 vs 2.5\%), whereas photosensitivity was seen less frequently (0.11 vs 3.5\%). Although in $3.0 \%$ of treatments $(137 / 4514)$, that is, in $6.9 \%$ of patients $(121 / 1755)$ there was a reported loss of more than 20 letters, in only two of these reports $(0.04 \%$ of treatments, $0.11 \%$ of patients) was the loss reported to have occurred within 7 days of treatment. However, it must be recognised the loss of 20 letters within 7 days may be under-reported since, in the absence of objective measurements at the time, it often relies upon patient information.

It is acknowledged that this surveillance programme was established without additional resources and relied heavily on clinicians volunteering to furnish data on regular basis in addition to their busy clinical practices. Data submission was largely paper based, posted, and collected in Liverpool and manually entered in electronic database. This paper demonstrates that multicentre data collection is possible within an established network that can provide relevant clinical information. However, to improve the efficiency of future sureveillance programmes, appropriate resources should be made available to the participating centres.

\section{Conclusion}

The results of the PDTUG surveillance programme demonstrate that the response to verteporfin PDT reported in the treatment arm of the TAP study can be achieved in routine clinical practice in a mixed lesion population and with a good safety profile. Our findings are important and reassuring to health service commissioners when considering the generalisability of the research study results of verteporfin PDT into routine clinical practice, at least in recognised centres of retinal expertise.

\section{Acknowledgements}

The database development and data extraction was supported by an unrestricted grant from Novartis Pharma AG.

\section{References}

1 Ferris III FL, Fine SL, Hyman L. Age-related macular degeneration and blindness due to neovascular maculopathy. Arch Ophthalmol 1984; 102: 1640-1642.

2 Bellmann C, Unnebrink K, Rubin GS, Miller D, Holz FG. Visual acuity and contrast sensitivity in patients with neovascular age-related macular degeneration. Results from the radiation therapy for age-related macular degeneration (rad-) study. Graefes Arch Clin Exp Ophthalmol 2003; 241: 968-974.

3 Meads C, Salas C, Roberts T, Moore D, Fry-Smith A, Hyde C. Clinical effectiveness and cost-utility of photodynamic therapy for wet age-related macular degeneration: a systematic review and economic evaluation. Health Technol Assess 2003; 7: 1-98.

4 Starr CE, Guyer DR, Yannuzzi LA. Age-related macular degeneration. Can we stem this worldwide public health crisis? Postgrad Med 1998; 103: 153-156, 161-164. 
5 Evans JR, Fletcher AE, Wormald RPL. Age-related macular degeneration causing visual impairment in people 75 years or older in Britain: an add-on study to the Medical Research Council Trial of Assessment and Management of Older People in the Community. Ophthalmology 2004; 111: 513-517.

6 Owen CG, Fletcher AE, Donoghue M, Rudnicka AR. How big is the burden of visual loss caused by age related macular degeneration in the United Kingdom? $\mathrm{Br} \mathrm{J}$ Ophthalmol 2003; 87: 312-317.

7 Monés J, Rubin GS. Contrast sensitivity as an outcome measure in patients with subfoveal choroidal neovascularisation due to age-related macular degeneration. Eye 2005; 19: 1142-1150.

8 Dong LM, Childs AL, Mangione CM, Bass EB, Bressler NM, Hawkins BS et al Health - and vision-related quality of life among patients with choroidal neovascularization secondary to age-related macular degeneration at enrollment in randomized trials of submacular surgery: SST report no. 4. Am J Ophthalmol 2004; 138: 91-108.

9 Fine SL, Berger JW, Maguire MG, Ho AC. Age-related macular degeneration. $N$ Engl J Med 2000; 342: 483-492.

10 Photodynamic therapy of subfoveal choroidal neovascularization in age-related macular degeneration with verteporfin: one-year results of 2 randomized clinical trials - TAP Report 1. Treatment of age-related macular degeneration with photodynamic therapy (TAP) Study Group. Arch Ophthalmol 1999; 117: 1329-1345.

11 Frennesson CI, Nilsson SEG. Encouraging results of photodynamic therapy with Visudyne in a clinical patient material of age-related macular degeneration. Acta Ophthalmol Scand 2004; 82: 645-650.

12 Bressler NM. Treatment of Age-Related Macular Degeneration with Photodynamic Therapy (TAP) Study Group Photodynamic therapy of subfoveal choroidal neovascularization in age-related macular degeneration with verteporfin. Two-year results of 2 randomized clinical trials - TAP Report 2. Arch Ophthalmol 2001; 119: 198-207.

13 Verteporfin in Photodynamic Therapy (VIP) Study Group. Photodynamic therapy of subfoveal choroidal neovascularization in pathologic myopia with verteporfin: 1-year results of a randomized clinical trial - VIP Report No. 1. Ophthalmology 2001; 108: 841-852.

14 Verteporfin in Photodynamic Therapy Study Group. Verteporfin therapy of subfoveal choroidal neovascularization in age-related macular degeneration: two-year results of a randomized clinical trial including lesions with occult with no classic choroidal neovascularization - verteporfin in photodynamic therapy report 2. Am J Ophthalmol 2001; 131: 541-560.
15 National Institute for Clinical Excellence. Guidance on the use of photodynamic therapy for age-related macular degeneration, September 2003.

16 Experimental Ophthalmology Unit: age-related macular degeneration. Photodynamic therapy for AMD - information for health authorities and purchasers, The University of Liverpool websitewww.liv.ac.uk/ophthalmology/ pdtinfo2.htm.

\section{Appendix \\ Clinical centres and investigators in the PDTUG surveillance programme}

Barnet Hospital, Barnet, Herts (BAR): principal investigator - $\mathrm{M}$ Harris;

Royal United Hospital, Bath (BAT): principal investigator - R Antcliff; Bradford Teaching Hospitals, Royal Infirmary, Bradford (BRD): principal investigator FD Ghanchi, Co-investigators: J Dixon, S Bell: Winterbourne Hospital, Dorchester (DOR): principal investigator - AC Reck, co-investigators - C Young, J Chappell, B Jennings, S Coombs; Diana Princess of Wales Hospital, Grimsby (GRI): principal investigator S Kotta; St James' University Hospital, Leeds (LEE): principal investigator - M McKibbin, co-investigators: K West, T Hunter, A Jones, M Stockton; St Paul's Eye Unit, Royal Liverpool University Hospital (LIV): principal investigator: SP Harding; co-investigators: MC Briggs, IA Pearce, PM Lenfestey, A Tompkin, V Tompkin, T Turpin, SA Taylor, L Gee; Manchester Royal Eye Hospital (MAN): principal investigator - P Bishop; Department of Ophthalmology, Royal Victoria Hospital,

Newcastle-upon-Tyne (NEW): principal investigator - SJ Talks, co-investigators - R Kak, B Hope, K Gales, H Harris; Royal Hallamshire Hospital, Sheffield (SHE): principal investigator - C Brand, co-investigators: M Freeman, E Watts, C Mody, G Simpson; Sunderland Eye Infirmary (SUN): principal investigator - D Steel; Department of Ophthalmology, Torbay Hospital, Torquay (TOR): principal investigator - MD Cole, co-investigators - Y Osoba, L Walker, A Field, R Willox, P Ladwa, G Pearson; Wolverhampton and Midland Counties Eye Infirmary (WLV): principal investigator YC Yang. 